\title{
A DEGENERATE NEUMANN PROBLEM FOR QUASILINEAR ELLIPTIC INTEGRO-DIFFERENTIAL OPERATORS
}

\author{
D. K. Palagachev, P. R. Popivanov and K. Taira
}

ABstract. This paper is devoted to the study of the following degenerate Neumann problem for a quasilinear elliptic integro-differential operator

$$
\begin{cases}\mathcal{W} u(x)=f(x, u, D u) & \text { in } \Omega, \\ \mathcal{L} u(x)=\varphi(x) & \text { on } \Gamma\end{cases}
$$

Here $\mathcal{W}$ is a second-order elliptic integro-differential operator of Waldenfels type and $\mathcal{L} u=a(x) \frac{\partial u}{\partial \boldsymbol{\nu}}+b(x) u$ is a first-order Ventcel' operator with $a(x)$ and $b(x)$ being nonnegative smooth functions on $\Gamma$ such that $a(x)+b(x)>0$ on $\Gamma$. Classical existence and uniqueness results in the framework of Hölder spaces are derived under suitable regularity and structure conditions on the nonlinear term $f(x, u, D u)$.

\section{Introduction and Main Results}

This paper is a continuation of the previous note [11] where we studied a degenerate Neumann problem for quasilinear second-order elliptic differential operators and proved classical existence and uniqueness results in the framework of Hölder spaces under suitable regularity and structure conditions on the nonlinear term. The purpose of this paper is to extend these results to the integro-differential operator case.

Let $\Omega$ be a bounded and convex domain of Euclidean space $\mathbb{R}^{n}, n \geq 2$, with smooth boundary $\Gamma$; its closure $\bar{\Omega}=\Omega \cup \Gamma$ is an $n$-dimensional, compact smooth manifold with boundary.

Let $\mathcal{W}$ be a second-order, elliptic integro-differential operator with real coefficients such that

$$
\begin{aligned}
\mathcal{W} u(x)= & \mathcal{A} u(x)+\mathcal{S} u(x) \\
:= & \sum_{i, j=1}^{n} a^{i j}(x) \frac{\partial^{2} u}{\partial x_{i} \partial x_{j}}(x) \\
& +\int_{\mathbb{R}^{n} \backslash\{0\}}\left(u(x+z)-u(x)-\sum_{j=1}^{n} z_{j} \frac{\partial u}{\partial x_{j}}(x)\right) s(x, z) m(d z) .
\end{aligned}
$$

Here:

1991 Mathematics Subject Classification. 35J65, 35R25.

Key words and phrases. Quasilinear elliptic integro-differential operator, degenerate Neumann problem, a'priori estimate, maximum and comparison principles. 
(1) $a^{i j} \in C^{\infty}(\bar{\Omega}), a^{i j}(x)=a^{j i}(x)$ and there exists a constant $a_{0}>0$ such that

$$
\sum_{i, j=1}^{n} a^{i j}(x) \xi_{i} \xi_{j} \geq a_{0}|\xi|^{2}, \quad x \in \bar{\Omega}, \xi \in \mathbb{R}^{n}
$$

(2) $s(x, z) \in C\left(\bar{\Omega} \times \mathbb{R}^{n}\right)$ and $0 \leq s(x, z) \leq 1$ in $\bar{\Omega} \times \mathbb{R}^{n}$, and there exist constants $C_{0}>0$ and $0<\theta_{0}<1$ such that

$$
|s(x, z)-s(y, z)| \leq C_{0}|x-y|^{\theta_{0}}, \quad x, y \in \bar{\Omega}, z \in \mathbb{R}^{n}
$$

and

$$
s(x, z)=0 \quad \text { if } x+z \notin \bar{\Omega} .
$$

Condition (1.1) implies that the integral operator $\mathcal{S}$ may be considered as an operator acting on functions $u$ defined on the closure $\bar{\Omega}$.

(3) The measure $m(d z)$ is a Radon measure on $\mathbb{R}^{n} \backslash\{0\}$ which satisfies the moment condition

$$
\int_{\{|z| \leq 1\}}|z|^{2} m(d z)+\int_{\{|z|>1\}}|z| m(d z)<\infty .
$$

The operator $\mathcal{W}=\mathcal{A}+\mathcal{S}$ is called a second-order Waldenfels operator. The differential operator $\mathcal{A}$ is called a diffusion operator which describes analytically a strong Markov process with continuous paths in the interior $\Omega$ such as Brownian motion. The integral operator $\mathcal{S}$ is called a second-order Lévy operator which is supposed to correspond to the jump phenomenon in the closure $\bar{\Omega}$ (see [2], [7], [8]). In this context, condition (1.1) implies that any Markovian particle does not move by jumps from $x \in \Omega$ to the outside of $\bar{\Omega}$.

Let $\mathcal{L}$ be a first-order, boundary operator with real coefficients such that

$$
\mathcal{L} u(x)=a(x) \frac{\partial u}{\partial \boldsymbol{\nu}}(x)+b(x) u(x) .
$$

Here:

(1) $a(x) \in C^{\infty}(\Gamma)$ and $a(x) \geq 0$ on $\Gamma$.

(2) $b(x) \in C^{\infty}(\Gamma)$ and $b(x) \geq 0$ on $\Gamma$.

(3) $\boldsymbol{\nu}$ is the unit exterior normal to $\Gamma$.

The boundary operator $\mathcal{L}$ is called a first-order Ventcel' boundary operator. The terms $a \partial u / \partial \boldsymbol{\nu}$ and $b u$ of $\mathcal{L}$ are supposed to correspond to the reflection phenomenon and the absorption phenomenon, respectively (see [2], [8]).

In this paper we study the following quasilinear elliptic boundary value problem: Given a continuous function $f(x, z, p)$ defined on $\bar{\Omega} \times \mathbb{R} \times \mathbb{R}^{n}$, find a function $u(x)$ in $\Omega$ such that

$$
\begin{cases}\mathcal{W} u(x)=f(x, u, D u) & \text { in } \Omega \\ \mathcal{L} u(x)=0 & \text { on } \Gamma .\end{cases}
$$

Here $D u$ stands for the gradient $\left(\partial u / \partial x_{1}, \partial u / \partial x_{2}, \ldots, \partial u / \partial x_{n}\right)$ of $u$. The interest to the problems of type (1.2) is prompted by their importance in the theory of 
stochastic processes. The nonlinear term $f(x, u, D u)$ represents such a branching phenomenon as in Brownian motion (cf. [5]).

The linear problem

$$
\begin{cases}\mathcal{W} u(x)=f(x) & \text { in } \Omega \\ \mathcal{L} u(x)=\varphi(x) & \text { on } \Gamma\end{cases}
$$

was studied by Taira [10] in the framework of Hölder spaces. The first purpose of this paper is to extend the existence and uniqueness result [10, Theorem 1] to the quasilinear problem (1.2).

Now we formulate our fundamental hypotheses on the nonlinear term $f(x, u, D u)$ :

Regularity conditions:

$$
\left\{\begin{array}{l}
f(x, z, p) \in C^{\theta_{0}}\left(\bar{\Omega} \times \mathbb{R} \times \mathbb{R}^{n}\right), \quad 0<\theta_{0}<1 \\
f(x, z, p) \text { is continuously differentiable with respect to } p .
\end{array}\right.
$$

Monotonicity condition:

$$
\left\{\begin{array}{l}
f(x, z, p) \text { is a monotone increasing and differentiable function } \\
\text { with respect to } z \in \mathbb{R} \text { for any }(x, p) \in \bar{\Omega} \times \mathbb{R}^{n} \text { and there exist } \\
\text { constants } f_{0}>0 \text { and } M_{1}>0 \text { such that } \\
\frac{\partial f}{\partial z}(x, z, p) \geq f_{0} \text { for any }(x, p) \in \bar{\Omega} \times \mathbb{R}^{n} \text { and } z \in \mathbb{R} \text { with }|z| \geq M_{1} .
\end{array}\right.
$$

Quadratic gradient growth condition: one can find a positive and non-decreasing function $f_{1}(t)$ on the interval $[0, \infty)$ such that

$$
|f(x, z, p)| \leq f_{1}(|z|)\left(1+|p|^{2}\right) \quad \text { for all }(x, z, p) \in \bar{\Omega} \times \mathbb{R} \times \mathbb{R}^{n} .
$$

Our final hypothesis concerns the behavior of the functions $a$ and $b$ on $\Gamma$ :

$$
a(x) \geq 0, b(x) \geq 0, a(x)+b(x)>0 \text { for all } x \in \Gamma .
$$

We should point out that problem (1.2) is a singular boundary value problem in view of condition (1.6), since the so-called Shapiro-Lopatinskii complementary condition is violated at the points $x \in \Gamma$ where $a(x)=0$.

Our main result is the following existence theorem of classical solutions in the framework of Hölder spaces:

Theorem 1.1. Suppose that conditions (1.3) through (1.6) are satisfied. Then the homogeneous problem (1.2) admits a unique classical solution $u \in C^{2+\theta}(\bar{\Omega})$ for each $\theta$ satisfying $0<\theta<\theta_{0}$.

The second purpose of this paper is to extend Theorem 1.1 to the non-homogeneous case. To do so, following Taira [9] and [10], we introduce the next interpolation Banach space

$$
C_{*}^{1+\theta}(\Gamma)=\left\{\varphi=a(x) \varphi_{1}+b(x) \varphi_{2}: \varphi_{1} \in C^{1+\theta}(\Gamma), \varphi_{2} \in C^{2+\theta}(\Gamma)\right\}, \quad 0<\theta<1,
$$

equipped with the norm

$$
\|\varphi\|_{C_{*}^{1+\theta}(\Gamma)}=\inf \left\{\left\|\varphi_{1}\right\|_{C^{1+\theta}(\Gamma)}+\left\|\varphi_{2}\right\|_{C^{2+\theta}(\Gamma)}: \varphi=a(x) \varphi_{1}+b(x) \varphi_{2}\right\} .
$$

We note that the space $C_{*}^{1+\theta}(\Gamma)$ is an "interpolation space" between $C^{2+\theta}(\Gamma)$ and $C^{1+\theta}(\Gamma)$; more precisely, it is easy to see that

$$
\begin{cases}C_{*}^{1+\theta}(\Gamma)=C^{2+\theta}(\Gamma) & \text { if } a(x) \equiv 0 \text { on } \Gamma, \\ C_{*}^{1+\theta}(\Gamma)=C^{1+\theta}(\Gamma) & \text { if } a(x)>0 \text { on } \Gamma .\end{cases}
$$

Then we have the following generalization of [11, Theorem 1.1] to the integrodifferential operator case: 
Theorem 1.2. Suppose that conditions (1.3) through (1.6) are satisfied. Then the non-homogeneous problem

$$
\begin{cases}\mathcal{W} u(x)=f(x, u, D u) & \text { in } \Omega \\ \mathcal{L} u(x)=\varphi(x) & \text { on } \Gamma\end{cases}
$$

admits a unique classical solution $u \in C^{2+\theta}(\bar{\Omega})$ for any $\varphi \in C_{*}^{1+\theta}(\Gamma)$.

The monotonicity condition (1.4) will play an important role in the proof of uniqueness of solutions of problems (1.2) and (1.7), and also in the proof of an a'priori estimate for the $C(\bar{\Omega})$-norm of these solutions. It turns out that, in some special cases we may replace the requirement on differentiability of $f(x, z, p)$ with respect to $z$ by a weaker structure condition. Thus, instead of condition (1.4) we impose the following monotonicity condition:

$$
\left\{\begin{array}{l}
f(x, z, p) \text { is a monotone increasing function with respect } \\
\text { to its second argument } z \in \mathbb{R} \text { for any }(x, p) \in \bar{\Omega} \times \mathbb{R}^{n} \text {, and } \\
\text { there exists a constant } M_{2}>0 \text { such that } \\
\text { sign } z \cdot f(x, z, 0) \geq 0 \text { for any } x \in \bar{\Omega} \text { and } z \in \mathbb{R} \text { with }|z| \geq M_{2} .
\end{array}\right.
$$

Then Theorems 1.1 and 1.2 can be generalized as follows:

Theorem 1.3. If conditions (1.3), (1.5), (1.6) and (1.8) are satisfied, then we have the following:

(i) The homogeneous problem (1.2) possesses a unique classical solution $u \in$ $C^{2+\theta}(\bar{\Omega})$ for each $\theta$ satisfying $0<\theta<\theta_{0}$.

(ii) In addition to the above requirements, suppose that $b(x)>0$ on $\Gamma$. Then the non-homogeneous problem (1.7) is uniquely solvable in the space $C^{2+\theta}(\bar{\Omega})$ for any $\varphi \in C_{*}^{1+\theta}(\Gamma)$.

We give two simple examples for the function $f(x, z, p)$ :

Examples 1.4. (i) The function $f(x, z, p)=g(x) z^{2 k+1}\left(1+|p|^{2}\right)$ satisfies condition (1.4) if $g(x)>0$ on $\bar{\Omega}$. Here $k$ is a non-negative integer.

(ii) The function $f(x, z, p)=g(x) z e^{z}\left(1+|p|^{2}\right)$ satisfies condition (1.8) if $g(x) \geq 0$ on $\bar{\Omega}$.

The rest of the paper is organized as follows. In Section 2 we prove a comparison principle for quasilinear integro-differential operators (Lemma 2.1), and derive $C^{1+\theta}(\bar{\Omega})$-a'priori estimates for the solutions to problems (1.2) and (1.7) (Lemmas 2.2, 2.3 and 2.5). Section 3 is devoted to the proof of Theorems 1.1, 1.2 and 1.3. The uniqueness result is an immediate consequence of the comparison principle. The solvability of problems (1.2) and (1.7) follows from an application of the Leray-Schauder fixed point theorem, by making use of the a'priori estimates for the solutions $u \in C^{2+\theta}(\bar{\Omega})$ to problem (1.2) or problem (1.7).

Throughout the paper the letter $C$ will denote a generic positive constant independent of the solutions to the relative problem under consideration. Any such constant may vary from a line into another. 


\section{A'Priori Estimates}

In order to prove our results, we shall apply the Leray-Schauder fixed point theorem (see [4, Theorem 11.3]). So we must establish a'priori estimates for the $C^{1+\theta}(\bar{\Omega})$-norm of every solution $u \in C^{2+\theta}(\bar{\Omega})$ to problem (1.2) or problem (1.7).

To begin with, the following comparison principle for quasilinear operators will be established.

Lemma 2.1. Suppose that conditions (1.3) and (1.6) are fulfilled and that $f(x, z, p)$ is monotone increasing in $z$ for each $(x, p) \in \Omega \times \mathbb{R}^{n}$ and is differentiable with respect to $p$ for each $(x, z, p) \in \Omega \times \mathbb{R} \times \mathbb{R}^{n}$. If $u, v \in C^{2}(\Omega) \cap C^{1}(\bar{\Omega})$ satisfy the conditions

$$
\begin{cases}\mathcal{W} u(x)-f(x, u, D u) \geq \mathcal{W} v(x)-f(x, v, D v) & \text { in } \Omega \\ \mathcal{L} u(x) \leq \mathcal{L} v(x) & \text { on } \Gamma\end{cases}
$$

then we have $u(x) \leq v(x)$ on $\bar{\Omega}$.

Proof. Let $w=u-v$, and suppose to the contrary that the set

$$
\Omega^{+}=\{x \in \Omega: w(x)>0\}=\{x \in \Omega: u(x)>v(x)\}
$$

is non-empty. Then it follows that

$$
\mathcal{W} w(x)+f(x, u, D v)-f(x, u, D u) \geq f(x, u, D v)-f(x, v, D v)>0 \quad \text { on } \Omega^{+}
$$

since $f(x, z, p)$ increases with respect to its second argument $z$. Thus, by letting

$$
b^{i}(x)=-\int_{0}^{1} \frac{\partial f}{\partial p_{i}}(x, u(x), t D w(x)+D v(x)) d t
$$

we obtain that

$$
\mathcal{W} w(x)+\sum_{i=1}^{n} b^{i}(x) \frac{\partial w}{\partial x_{i}}(x)>0 \quad \text { on } \Omega^{+} .
$$

If $x_{0}$ is a point of $\bar{\Omega}$ such that $w\left(x_{0}\right)=\max _{\bar{\Omega}} w(x)>0$, then it follows from an application of the strong interior maximum principle [2, Théorème VII] that

$$
x_{0} \in \partial \Omega^{+} \cap \Gamma
$$

Therefore we have, by the boundary point lemma [2, Théorème VIII],

$$
\frac{\partial w}{\partial \boldsymbol{\nu}}\left(x_{0}\right)>0
$$

However it follows from condition (1.6) that

$$
\mathcal{L} w\left(x_{0}\right)=a\left(x_{0}\right) \frac{\partial w}{\partial \boldsymbol{\nu}}\left(x_{0}\right)+b\left(x_{0}\right) w\left(x_{0}\right)>0
$$

This contradicts the boundary condition $\mathcal{L} w(x) \leq 0$ on $\Gamma$.

Summing up, we have proved that the set $\Omega^{+}$is empty and the statement follows. 
2.1 A'priori estimate for $\|u\|_{C(\bar{\Omega})}$. As a first step, we establish an a'priori estimate for the norm $\|u\|_{C(\bar{\Omega})}$ of every solution $u \in C^{2+\theta}(\bar{\Omega})$ to the homogeneous problem (1.2):

Lemma 2.2. Suppose that conditions (1.3), (1.4) and (1.6) are fulfilled. If $u \in$ $C^{2}(\Omega) \cap C^{1}(\bar{\Omega})$ is a solution to problem (1.2), then we have

$$
\|u\|_{C(\bar{\Omega})}=\max _{\bar{\Omega}}|u(x)| \leq M_{1}+\frac{\max _{\bar{\Omega}}\left|f\left(x, M_{1}, 0\right)\right|}{f_{0}},
$$

where $M_{1}>0$ is the constant given in condition (1.4).

Proof. By letting

$$
K_{1}=M_{1}+\frac{\max _{\bar{\Omega}}\left|f\left(x, M_{1}, 0\right)\right|}{f_{0}},
$$

we obtain from condition (1.4) that

$$
\begin{aligned}
\mathcal{W} u(x)-f(x, u, D u)=0 \geq & \left(M_{1}-K_{1}\right) f_{0}-f\left(x, M_{1}, 0\right) \\
\geq & \left(M_{1}-K_{1}\right) \int_{0}^{1} \frac{\partial f}{\partial z}\left(x, t\left(K_{1}-M_{1}\right)+M_{1}, 0\right) d t \\
& -f\left(x, M_{1}, 0\right) \\
= & -f\left(x, K_{1}, 0\right)=\mathcal{W} K_{1}(x)-f\left(x, K_{1}, D K_{1}\right) \text { in } \Omega,
\end{aligned}
$$

and

$$
\mathcal{L} u(x)=0 \leq b(x) K_{1}=\mathcal{L} K_{1}(x) \quad \text { on } \Gamma .
$$

Therefore it follows from an application of Lemma 2.1 that $u(x) \leq K_{1}$ for each $x \in \bar{\Omega}$.

Repeating the same procedure with $u(x)$ replaced by $-u(x)$ and $f(x, z, p)$ replaced by $-f(x,-z,-p)$, we obtain that $-u(x) \leq K_{1}$ for each $x \in \bar{\Omega}$.

This completes the proof of Lemma 2.2.

The next result makes use of the monotonicity condition (1.8) instead of the monotonicity condition (1.4):

Lemma 2.3. Suppose that conditions (1.3), (1.6) and (1.8) are satisfied. Then we have the following:

(i) If $u \in C^{2}(\Omega) \cap C^{1}(\bar{\Omega})$ is a solution to the homogeneous problem (1.2), then we have the estimate

$$
\|u\|_{C(\bar{\Omega})} \leq M_{2}
$$

where $M_{2}>0$ is the constant given in condition (1.8).

(ii) Furthermore suppose that $b(x)>0$ on $\Gamma$. If $u \in C^{2}(\Omega) \cap C^{1}(\bar{\Omega})$ is a solution to the non-homogeneous problem (1.7), then we have, with $b_{0}=\min _{\Gamma} b(x)>0$,

$$
\|u\|_{C(\bar{\Omega})} \leq M_{2}+\frac{\max _{\Gamma}|\varphi(x)|}{b_{0}}
$$

Proof. (i) If $u$ solves the homogeneous problem (1.2), it follows from condition (1.8) that

$$
\mathcal{W} M_{2}(x)-f\left(x, M_{2}, D M_{2}\right)=-f\left(x, M_{2}, 0\right) \leq 0 \quad \text { in } \Omega
$$


Hence we have

$$
\mathcal{W} u(x)-f(x, u, D u)=0 \geq \mathcal{W} M_{2}(x)-f\left(x, M_{2}, D M_{2}\right) \quad \text { in } \Omega
$$

and also

$$
\mathcal{L} u(x)=0 \leq b(x) M_{2}=\mathcal{L} M_{2}(x) \quad \text { on } \Gamma .
$$

Therefore, by applying Lemma 2.1 we obtain that $|u(x)| \leq M_{2}$.

(ii) If $u$ solves the non-homogeneous problem (1.7), we let

$$
K_{2}=M_{2}+\frac{\max _{\Gamma}|\varphi(x)|}{b_{0}}
$$

Then we have, just as in part (i),

$$
\begin{aligned}
\mathcal{W} u(x)-f(x, u, D u)=0 & \geq-f\left(x, K_{2}, 0\right) \\
& =\mathcal{W} K_{2}(x)-f\left(x, K_{2}, D K_{2}\right) \quad \text { in } \Omega
\end{aligned}
$$

and also

$$
\begin{aligned}
\mathcal{L} u(x)=\varphi(x) \leq \max _{\Gamma}|\varphi(x)| & \leq b(x) \frac{\max _{\Gamma}|\varphi(x)|}{b_{0}} \\
& \leq b(x) K_{2}=\mathcal{L} K_{2}(x) \quad \text { on } \Gamma
\end{aligned}
$$

Hence it follows from an application of Lemma 2.1 that $|u(x)| \leq K_{2}$.

The proof of Lemma 2.3 is complete.

2.2 A'priori estimate for $[u]_{C^{1+\theta}(\bar{\Omega})}$. After having the a'priori estimate (2.1), the desired bound on $\|u\|_{C^{1+\theta}(\bar{\Omega})}$ will follow immediately if we have a uniform estimate for the Hölder seminorm

$$
[u]_{C^{1+\theta}(\bar{\Omega})}:=[D u]_{C^{\theta}(\bar{\Omega})}=\sup _{x, y \in \Omega} \frac{|D u(x)-D u(y)|}{|x-y|^{\theta}} .
$$

On the other hand, the Morrey lemma assures the imbedding of the Sobolev space $W^{2, p}(\Omega)$ into $C^{1+\theta}(\bar{\Omega})$ with $p=n /(1-\theta)$. Therefore the bound on $[D u]_{C^{\theta}(\bar{\Omega})}$ becomes equivalent to a uniform (with respect to $u$ ) estimate for the Sobolev norm $\|u\|_{W^{2, p}(\Omega)}$ of every solution to problem (1.2). According to [10, Theorem 3] (see Proposition 2.4 below also) and condition (1.5), we obtain that

$$
\|u\|_{W^{2, p}(\Omega)} \leq C f_{1}\left(\|u\|_{C(\bar{\Omega})}\right)\left(1+\|D u\|_{L^{2 p}(\Omega)}^{2}\right) .
$$

In order to estimate the norm $\|D u\|_{L^{2 p}(\Omega)}^{2}$, we shall use an approach due to AmannCrandall [1]. In this way, we will conclude that there exists a non-negative and increasing function $\gamma(t)$ on the interval $[0, \infty)$, which depends only on known quantities, such that

$$
\|u\|_{W^{2, p}(\Omega)} \leq \gamma\left(\|u\|_{C(\bar{\Omega})}\right)
$$

and hence the desired bound on $[u]_{C^{1+\theta}(\bar{\Omega})}$ will follow from an application of the Morrey lemma.

For the later purposes, we need the following version of [10, Theorem 3.1]: 
Proposition 2.4. There exists a constant $\lambda>0$ such that the linear problem

$$
\begin{cases}\mathcal{W} u(x)-\lambda u(x)=g(x) & \text { in } \Omega, \\ \mathcal{L} u(x)=0 & \text { on } \Gamma\end{cases}
$$

admits a unique strong solution $u \in W^{2, p}(\Omega)$ for any $g \in L^{p}(\Omega)$. Moreover we have the estimate

$$
\|u\|_{W^{2, p}(\Omega)} \leq C\|g\|_{L^{p}(\Omega)},
$$

with a constant $C>0$ independent of the solution $u$.

To proceed further, we fix a constant $\lambda>0$ as in Proposition 2.4. The next lemma is an essential step in the proof of the a'priori estimate for the seminorm $[u]_{C^{1+\theta}(\bar{\Omega})}$ of every solution $u \in C^{2+\theta}(\bar{\Omega})$ to the homogeneous problem (1.2):

Lemma 2.5. Let $p=n /(1-\theta)$ and suppose that conditions (1.3) through (1.6) are fulfilled. Then there exists a constant $C>0$, depending on the data of problem (1.2) and on $\|u\|_{C(\bar{\Omega})}$, such that

$$
\|D u\|_{L^{2 p}(\Omega)} \leq C
$$

for every solution $u \in C^{2+\theta}(\bar{\Omega})$ to problem (1.2).

Proof. (I) Let $u \in C^{2+\theta}(\bar{\Omega})$ be an arbitrary solution to problem (1.2), and let

$$
\begin{aligned}
& B(x)=-\frac{f(x, u(x), D u(x))}{1+|D u(x)|^{2}}, \\
& F(x)=\frac{f(x, u(x), D u(x))}{1+|D u(x)|^{2}}-\lambda u(x),
\end{aligned}
$$

just as in Amann-Crandall [1]. Then it follows that $B(x), F(x) \in C^{\theta}(\bar{\Omega})$. Hence the function $u(x)$ solves the problem

$$
\begin{cases}\mathcal{W} u(x)+B(x)|D u(x)|^{2}-\lambda u(x)=F(x) & \text { in } \Omega, \\ \mathcal{L} u(x)=0 & \text { on } \Gamma .\end{cases}
$$

Now, for the fixed function $u(x)$, we imbed problem (2.4) into one-parameter family of problems

$$
\begin{cases}\mathcal{W} u(x ; \sigma)+B(x)\left|D_{x} u(x ; \sigma)\right|^{2}-\lambda u(x ; \sigma)=\sigma F(x) & \text { a.e. in } \Omega, \\ \mathcal{L} u(x ; \sigma)=0 & \text { on } \Gamma,\end{cases}
$$

depending on the parameter $\sigma \in[0,1]$.

(II) Our aim will be to study problem (2.5) in the framework of Sobolev spaces $W^{2, p}(\Omega)$ with $p=n /(1-\theta)$. Indeed, at this stage of our investigation, we do not know any existence or uniqueness result concerning problem (2.5). However it should be noted that, setting $\sigma=0$, problem (2.5) has at least one solution and it is the trivial one. Further on, problem (2.5) with $\sigma=1$ coincides with the original problem (1.2) and an eventual uniqueness result would imply that $u(x ; 1) \equiv u(x)$. The strategy we are going to follow consists of estimating $\left\|D_{x} u\left(x ; \sigma_{2}\right)\right\|_{L^{2 p}(\Omega)}$ in terms of $\left\|D_{x} u\left(x ; \sigma_{1}\right)\right\|_{L^{2 p}(\Omega)}$ whenever the difference $\sigma_{2}-\sigma_{1}>0$ is sufficiently small. Then, an additional solvability result for problem $(2.5)$ in the space $W^{2, p}(\Omega)$ for each $\sigma \in[0,1]$ would assure estimate $(2.2)$ by iteration of the norms $\left\|D_{x} u(x ; \sigma)\right\|_{L^{2 p}(\Omega)}$ for $\sigma<1$.

(II-a) We start to realize our aim by inferring the continuous dependence of problem (2.5) on the parameter $\sigma$ : 
Proposition 2.6. Let $u\left(x ; \sigma_{1}\right), u\left(x ; \sigma_{2}\right) \in W^{2, p}(\Omega)$ solve problem (2.5) with corresponding parameters $\sigma_{1} \leq \sigma_{2}$. Then we have the inequality

$$
\left\|u\left(x ; \sigma_{1}\right)-u\left(x ; \sigma_{2}\right)\right\|_{C(\bar{\Omega})} \leq\left(\sigma_{2}-\sigma_{1}\right) \widetilde{f}_{\lambda}\left(\|u\|_{C(\bar{\Omega})}\right)
$$

where $\widetilde{f}_{\lambda}(t)$ is a positive and non-decreasing function on the interval $[0, \infty)$ defined by the formula

$$
\tilde{f}_{\lambda}(t)=\frac{f_{1}(t)+\lambda t}{\lambda}
$$

Proof. The difference

$$
v(x)=u\left(x ; \sigma_{1}\right)-u\left(x ; \sigma_{2}\right) \in W^{2, p}(\Omega)
$$

solves the linear problem

$$
\begin{cases}\mathcal{W} v(x)+\sum_{i=1}^{n} B^{i}(x) \frac{\partial v}{\partial x_{i}}(x)-\lambda v(x)=\left(\sigma_{1}-\sigma_{2}\right) F(x) & \text { a.e. in } \Omega \\ \mathcal{L} v(x)=0 & \text { on } \Gamma,\end{cases}
$$

where

$$
B^{i}(x)=2 B(x) \int_{0}^{1}\left(t \frac{\partial v}{\partial x_{i}}(x)+\frac{\partial u}{\partial x_{i}} u\left(x ; \sigma_{2}\right)\right) d t \in C^{\theta}(\bar{\Omega}) .
$$

Therefore we have, by condition (1.5) and Lemma 2.2,

$$
\|v\|_{C(\bar{\Omega})} \leq\left(\sigma_{2}-\sigma_{1}\right) \tilde{f}_{\lambda}\left(\|u\|_{C(\bar{\Omega})}\right)
$$

Indeed, in order to apply Lemma 2.2 we must show that $v \in C^{2}(\Omega) \cap C^{1}(\bar{\Omega})$, while we only know that $v \in W^{2, p}(\Omega)$. However, standard bootstrapping arguments infer that $v \in C^{2}(\Omega) \cap C^{1}(\bar{\Omega})$. In fact, the function $v$ solves the problem

$$
\begin{cases}\mathcal{W} v(x)-\lambda v(x)=\left(\sigma_{1}-\sigma_{2}\right) F(x)-\sum_{i=1}^{n} B^{i}(x) \frac{\partial v}{\partial x_{i}}(x) & \text { a.e. in } \Omega, \\ \mathcal{L} v(x)=0 & \text { on } \Gamma,\end{cases}
$$

with

$$
\left(\sigma_{1}-\sigma_{2}\right) F(x)-\sum_{i=1}^{n} B^{i}(x) \frac{\partial v}{\partial x_{i}}(x) \in C^{\theta}(\bar{\Omega}) .
$$

Hence it follows that $v \in C^{2+\theta}(\bar{\Omega})$, since the mappings $\mathcal{W}-\lambda I: W^{2, p}(\Omega) \rightarrow L^{p}(\Omega)$ and $\mathcal{W}-\lambda I: C^{2+\theta}(\bar{\Omega}) \rightarrow C^{\theta}(\bar{\Omega})$ are both bijective (see Proposition 2.4 and [10, Theorem 1]).

Corollary 2.7. Problem (2.5) has at most one solution $u(x ; \sigma) \in W^{2, p}(\Omega)$ for each $\sigma \in[0,1]$.

Proof. The statement is a trivial consequence of estimate (2.6) if we take $\sigma_{1}=\sigma_{2}$ therein. 
(II-b) To proceed further with the proof of Lemma 2.5, let $\sigma_{1}<\sigma_{2}$ be two arbitrary values of the parameter $\sigma \in[0,1]$ and let $u\left(x ; \sigma_{1}\right), u\left(x ; \sigma_{2}\right) \in W^{2, p}(\Omega)$ be the corresponding solutions to problem (2.5). Then it follows that the difference

$$
v(x)=u\left(x ; \sigma_{1}\right)-u\left(x ; \sigma_{2}\right) \in W^{2, p}(\Omega)
$$

is a solution to the problem

$$
\left\{\begin{array}{rlrl}
\mathcal{W} v(x) & -\lambda v(x)=\left(\sigma_{1}-\sigma_{2}\right) F(x) & \\
& -B(x)\left(\left|D_{x} u\left(x ; \sigma_{1}\right)\right|^{2}-\left|D_{x} u\left(x ; \sigma_{2}\right)\right|^{2}\right) & & \text { a.e. in } \Omega \\
\mathcal{L} v(x)=0 & & \text { on } \Gamma .
\end{array}\right.
$$

Hence it follows from an application of Proposition 2.4 that

$$
\|v\|_{W^{2, p}(\Omega)} \leq C\left\|\left(\sigma_{1}-\sigma_{2}\right) F-B\left(\left|D_{x} u\left(\cdot ; \sigma_{1}\right)\right|^{2}-\left|D_{x} u\left(\cdot ; \sigma_{2}\right)\right|^{2}\right)\right\|_{L^{p}(\Omega)}
$$

so that, in view of condition (1.5), definitions (2.3) and estimate (2.1)

$$
\|v\|_{W^{2, p}(\Omega)} \leq C\left(1+\|D v\|_{L^{2 p}(\Omega)}^{2}+\left\|D_{x} u\left(\cdot ; \sigma_{1}\right)\right\|_{L^{2 p}(\Omega)}^{2}\right) .
$$

However, by combining the Gagliardo-Nirenberg inequality (see [3], [6]) and inequality (2.6) we can estimate the second term on the right of inequality (2.7) as follows:

$$
\begin{aligned}
\|D v\|_{L^{2 p}(\Omega)}^{2} & \leq C\left\|D^{2} v\right\|_{L^{p}(\Omega)}\|v\|_{L^{\infty}(\Omega)} \\
& \leq C\left(\sigma_{2}-\sigma_{1}\right)\|v\|_{W^{2, p}(\Omega)} \widetilde{f}_{\lambda}\left(\|u\|_{C(\bar{\Omega})}\right) .
\end{aligned}
$$

Therefore we have, with a constant $C_{1}>0$ independent of $\sigma_{2}-\sigma_{1}$,

$$
\|D v\|_{L^{2 p}(\Omega)}^{2} \leq C_{1}\left(1+\left(\sigma_{2}-\sigma_{1}\right)\|D v\|_{L^{2 p}(\Omega)}^{2}+\left\|D_{x} u\left(\cdot ; \sigma_{1}\right)\right\|_{L^{2 p}(\Omega)}^{2}\right) .
$$

In particular, taking an $\varepsilon>0$ so small that $C_{1} \varepsilon<1 / 2$ we derive that

$$
\left\|D_{x} v\left(\cdot ; \sigma_{2}\right)\right\|_{L^{2 p}(\Omega)}^{2} \leq C_{2}+C_{3}\left\|D_{x} u\left(\cdot ; \sigma_{1}\right)\right\|_{L^{2 p}(\Omega)}^{2},
$$

whenever $\sigma_{2}-\sigma_{1} \leq \varepsilon$. Here $C_{2}>0, C_{3}>0$ are some constants. On the other hand, Corollary 2.7 tells us that $u\left(x ; \sigma_{1}\right) \equiv 0$ if $\sigma_{1}=0$.

Therefore, if we take $\sigma_{1}=0$ and $\sigma_{2}=\varepsilon$, we obtain from estimate (2.8) that

$$
\left\|D_{x} u(\cdot ; \varepsilon)\right\|_{L^{2 p}(\Omega)}^{2} \leq C_{2}
$$

for every solution $u(x ; \varepsilon) \in W^{2, p}(\Omega)$ to problem $(2.5)$ with $\sigma=\varepsilon$.

(III) In order to complete the proof of Lemma 2.5, it remains to prove the $W^{2, p}(\Omega)$ solvability of problem $(2.5)$ for $\sigma=\varepsilon$. We will make use of the LeraySchauder fixed point theorem. To do so, we introduce a compact nonlinear operator

$$
\mathcal{T}: W^{1,2 p}(\Omega) \longrightarrow W^{2, p}(\Omega) \underset{\text { compactly }}{\hookrightarrow} W^{1,2 p}(\Omega)
$$


as follows: For each function $w \in W^{1,2 p}(\Omega)$, the function $\mathcal{T} w \in W^{2, p}(\Omega)$ is the unique solution to the linear problem

$$
\begin{cases}\mathcal{W}(\mathcal{T} w)(x)-\lambda \mathcal{T} w(x)=\varepsilon F(x)-B(x)|D w(x)|^{2} & \text { a.e. in } \Omega \\ \mathcal{L}(\mathcal{T} w)(x)=0 & \text { on } \Gamma .\end{cases}
$$

Here we note that $|D w|^{2} \in L^{p}(\Omega)$ and that problem (2.10) is uniquely solvable in the space $W^{2, p}(\Omega)$ as a consequence of Proposition 2.4. The same result ensures the continuity of the mapping $\mathcal{T}$, while the Rellich theorem implies that $\mathcal{T}$ is a compact operator considered as a mapping of $W^{1,2 p}(\Omega)$ into itself. Further on, the bound (2.9) supplies a uniform (with respect to $w$ and $\tau$ ) a'priori estimate for every solution to the equation $w=\tau \mathcal{T} w, \tau \in[0,1]$, which is equivalent to the problem

$$
\begin{cases}\mathcal{W} w(x)-\lambda w(x)=\tau\left(\varepsilon F(x)-B(x)|D w(x)|^{2}\right) & \text { a.e. in } \Omega \\ \mathcal{L} w(x)=0 & \text { on } \Gamma .\end{cases}
$$

Therefore, by virtue of the Leray-Schauder fixed point theorem one can find a fixed point $w=\mathcal{T} w \in W^{1,2 p}(\Omega)$ of the mapping $\mathcal{T}, w \in W^{2, p}(\Omega)$ and it solves problem (2.5) with $\sigma=\varepsilon$.

(IV) Now we cover the interval $[0,1]$ by a finite number of intervals of length $\varepsilon$, and set $\sigma_{1}=m \varepsilon, \sigma_{2}=(m+1) \varepsilon, m=1,2, \ldots$, in estimate (2.8). The desired estimate $(2.2)$ for $u(x) \equiv u(x ; 1)$ follows by applying finitely many times the above procedure and using Corollary 2.7 at each step.

The proof of Lemma 2.5 is complete.

Bearing in mind Proposition 2.4, we derive the following:

Corollary 2.8. Under the assumptions of Lemmas 2.2 and 2.5, there exists a constant $C>0$, independent of $u$, such that

$$
\|u\|_{W^{2, p}(\Omega)} \leq C
$$

for every solution $u \in W^{2, p}(\Omega)$ to problem (1.2).

Corollary 2.9. If conditions (1.3) through (1.6) are fulfilled, then there exists a constant $C>0$, independent of $u$, such that

$$
\|u\|_{C^{1+\theta}(\bar{\Omega})} \leq C
$$

for every solution $u \in C^{2+\theta}(\bar{\Omega})$ to problem (1.2).

Proof. Estimate (2.11) is an immediate consequence of the Morrey lemma (i.e., $W^{2, p}(\Omega) \subset C^{1+\theta}(\bar{\Omega})$ with $\left.p=n /(1-\theta)\right)$ and Corollary 2.8.

\section{Proof of Main Results}

This section is devoted to the proof of Theorems 1.1, 1.2 and 1.3.

3.1 Proof of Theorem 1.1. The uniqueness assertion of Theorem 1.1 follows immediately from the monotonicity of $f(x, z, p)$ with respect to $z$ and the comparison principle (Lemma 2.1). 
In order to prove the solvability of problem (1.2), take an arbitrary function $v \in C^{1+\theta}(\bar{\Omega}), 0<\theta<\theta_{0}$, and consider the linear problem

$$
\begin{cases}\mathcal{W} u(x)=f(x, v, D v) & \text { in } \Omega, \\ \mathcal{L} u(x)=0 & \text { on } \Gamma .\end{cases}
$$

By virtue of condition (1.3), it follows that $f(x, v, D v) \in C^{\theta}(\bar{\Omega})$. Therefore $[10$, Theorem 1] asserts that problem (3.1) possesses a unique solution $u \in C^{2+\theta}(\bar{\Omega})$ for any $v \in C^{1+\theta}(\bar{\Omega})$. Define a nonlinear operator

$$
\mathcal{H}: C^{1+\theta}(\bar{\Omega}) \longrightarrow C^{2+\theta}(\bar{\Omega}) \underset{\text { compactly }}{\hookrightarrow \hookrightarrow} C^{1+\theta}(\bar{\Omega})
$$

by the formula $\mathcal{H} v=u$. Then it is easy to see that $\mathcal{H}$ is a compact operator from $C^{1+\theta}(\bar{\Omega})$ into itself. Further on, the continuity of $\mathcal{H}$ follows in a standard way from the fact that the operator $(\mathcal{W}, \mathcal{L})$ is a topological isomorphism between the Banach spaces $C^{2+\theta}(\bar{\Omega})$ and $C^{\theta}(\bar{\Omega}) \oplus\{0\}$ (see [10, Theorem 1]).

Now, for each $\rho \in[0,1]$ we consider the equation $u=\rho \mathcal{H} u$, that is, the problem

$$
\begin{cases}\mathcal{W} u(x)=\rho f(x, u, D u) & \text { in } \Omega, \\ \mathcal{L} u(x)=0 & \text { on } \Gamma .\end{cases}
$$

As it was already shown by Corollary 2.9, one can find a constant $C>0$, which depends only on the data of problem (3.2) but not on $u$ and $\rho$, such that

$$
\|u\|_{C^{1+\theta}(\bar{\Omega})} \leq C
$$

for every solution $u \in C^{2+\theta}(\bar{\Omega})$ to problem (3.2).

In this way, the properties of $\mathcal{H}$ and estimate (3.3) imply, through the LeraySchauder theorem, the existence of a fixed point $u \in C^{1+\theta}(\bar{\Omega})$ of the mapping $\mathcal{H}$. The function $u$ becomes a solution to problem (1.2) in view of the definition of $\mathcal{H}$. Finally, the smoothing properties of $\mathcal{H}$ yield that

$$
u=\mathcal{H} u \in C^{2+\theta}(\bar{\Omega}) .
$$

The proof of Theorem 1.1 is now complete.

3.2 Proof of Theorem 1.2. According to [10, Theorem 1], the linear problem

$$
\begin{cases}\mathcal{W} v(x)-v(x)=0 & \text { in } \Omega, \\ \mathcal{L} v(x)=\varphi(x) & \text { on } \Gamma\end{cases}
$$

possesses a unique classical solution $v \in C^{2+\theta}(\bar{\Omega})$. In this way, if $u$ is a solution to problem (1.7), then the function $w=u-v$ solves the homogeneous problem

$$
\begin{cases}\mathcal{W} w(x)=\bar{f}(x, w, D w) & \text { in } \Omega, \\ \mathcal{L} w(x)=0 & \text { on } \Gamma\end{cases}
$$

with

$$
\bar{f}(x, z, p)=f(x, z+v(x), p+D v(x))-v(x) .
$$

It is easy to verify that the function $\bar{f}(x, z, p)$ satisfies conditions of types (1.3) through (1.6), and we are in a position to apply Theorem 1.1. Indeed, it suffices to note that condition (1.4) is fulfilled by the function $\bar{f}(x, z, p)$ with the constant $M_{1}^{\prime}=M_{1}+\max _{\bar{\Omega}}|v(x)|$, and that estimate (2.11) remains valid for every solution $u \in C^{2+\theta}(\bar{\Omega})$ to the non-homogeneous problem (1.7), with some constant $C^{\prime}>0$ depending on $\|\varphi\|_{C_{*}^{1+\theta}(\Gamma)}$ in addition. 
3.3 Proof of Theorem 1.3. The proof of Theorem 1.3 may be carried out just as in the proof of Theorem 1.1 if we use Lemma 2.3 instead of Lemma 2.2. Furthermore, slight modifications should be done in the proof of the second statement of Theorem 1.3. Namely, one must consider one-parameter family of non-homogeneous boundary value problems

$$
\begin{cases}\mathcal{W} u(x ; \sigma)+B(x)\left|D_{x} u(x ; \sigma)\right|^{2}-\lambda u(x ; \sigma)=\sigma F(x) & \text { a.e. in } \Omega, \\ \mathcal{L} u(x ; \sigma)=\sigma \varphi(x) & \text { on } \Gamma,\end{cases}
$$

instead of problems (2.5) (cf. the proof of [11, Theorem 1.1]).

Acknowledgement. The research of the first author is supported by the Italian National Research Council (CNR) within the 1996 NATO Guest Fellowship Programme.

\section{REFERENCES}

1. H. Amann and M. Crandall, On some existence theorems for semi-linear elliptic equations, Indiana Univ. Math. J. 27 (1978), 779-790.

2. J.-M. Bony, P. Courrège et P. Priouret, Semi-groupes de Feller sur une varieté à bord compacte et problèmes aux limites intégro-différentiels du second ordre donnant lieu au principe du maximum, Ann. Inst. Fourier (Grenoble) 18 (1968), 369-521.

3. E. Gagliardo, Teoremi di esistenza e di unicità per problemi al contorno relativi ad equazioni paraboliche lineari e quasi lineari in $n$ variabili, Ricerche Mat. 5 (1956), 239-257.

4. D. Gilbarg and N. S. Trudinger, Elliptic partial differential equations of second order, second edition, Springer-Verlag, New York Berlin Heidelberg Tokyo, 1983.

5. T. E. Harris, The theory of branching processes, Springer-Verlag, Berlin Göttingen Heidelberg, 1963.

6. L. Nirenberg, On elliptic partial differential equations, Ann. Scuola Norm. Super. Pisa 13 (1959), 115-162.

7. D. W. Stroock, Diffusion processes associated with Lévy generators, Z. Wahrscheinlichkeitstheorie verw. Gebiete 32 (1975), 209-244.

8. K. Taira, On the existence of Feller semigroups with boundary conditions, Memoirs Amer. Math. Soc., Vol. 99, No. 475 (1992).

9. K. Taira, Analytic semigroups and semilinear initial boundary value problems, London Mathematical Society Lecture Note Series, No. 223, Cambridge University Press, Cambridge New York Melbourne, 1995.

10. K. Taira, Boundary value problems for elliptic integro-differential equations, Math. Z. 222 (1996), 305-327.

11. K. Taira, D. K. Palagachev and P. R. Popivanov, A degenerate Neumann problem for quasilinear elliptic equations, Tokyo J. Math. 23 (2000), 227-234.

Department of Mathematics, Technological University of Sofia, 8 "Kl. OkhridSKI" BlvD., 1756 SOFIA, BULGARIA

E-mail address: dian@bgcict.acad.bg

Institute of Mathematics, Bulgarian Academy of Sciences, "G. Bonchev" Str., BL. 8, 1113 SOFIA, BULGARIA

E-mail address: popivano@bgearn.acad.bg

Department of Mathematics, Hiroshima University, Higashi-Hiroshima 739-8526, JAPAN

E-mail address: taira@math.sci.hiroshima-u.ac.jp

Institute of Mathematics, University of Tsukuba, Tsukuba 305-8571, JAPAN

E-mail address: taira@math.tsukuba.ac.jp 